\title{
THE NOTION OF DIMENSION IN THE THEORY OF ALGEBRAIC DIFFERENTIAL EQUATIONS ${ }^{1}$
}

\author{
BY E. R. KOLCHIN \\ Communicated by L. Bers, March 18, 1964
}

Consider a system of algebraic differential equations

$$
P\left(y_{1}, \cdots, y_{n}\right)=0 \quad(P \in \Sigma)
$$

with coefficients in a differential field $\mathcal{F}$ (ordinary or partial); here $\Sigma$ is any subset of the differential polynomial algebra $Q=\mathcal{F}\left\{y_{1}, \cdots, y_{n}\right\}$ over $\mathcal{F}$. Denote the set of all solutions of this system by $3(\Sigma)$. We seek a measure of the size of $2(\Sigma)$. The analogous question for systems of algebraic equations (i.e. for affine algebraic geometry) has a satisfactory answer in the notion of dimension.

In the classical literature, where $\mathfrak{F}$ consists of meromorphic functions on some region of complex $m$-space, the solution is said to depend on a certain number $d$ of arbitrary functions of $m$ variables; if $d=0$ then the solution is said to depend on a certain number of arbitrary functions of $m-1$ variables; and so on. Of course, except in certain special cases, what this means (how these numbers are defined) is not made precise, and general results are therefore wanting.

The Ritt theory (see [1]) contains the beginning of a general answer to the question (when $F$ is of characteristic 0 ). First $\Sigma$ is replaced by the perfect differential ideal $\mathfrak{a}$ generated by $\Sigma$; this is harmless since $Z(\Sigma)=Z(\mathfrak{a})$. Then $\mathfrak{a}$ is expressed as the intersection of its components, $\mathfrak{a}=\mathfrak{p}_{1} \cap \cdots \cap \mathfrak{p}_{r}$; since $Z(\mathfrak{a})=Z\left(\mathfrak{p}_{1}\right) \cup \ldots \cup \mathfrak{Z}\left(\mathfrak{p}_{r}\right)$, the question is reduced to the case in which $\Sigma$ is a prime differential ideal $\mathfrak{p}$ of $\mathfrak{a}$. Finally, one takes a generic zero $\eta=\left(\eta_{1}, \cdots, \eta_{n}\right)$ of $\mathfrak{p}$, and computes the differential transcendence degree $d(p)$ of the differential field extension $\mathscr{F}\langle\eta\rangle$ of $\mathscr{F} ; d(\mathfrak{p})$ is called the differential dimension of $\mathfrak{p}$, or of $\mathfrak{Z}(\mathfrak{p})$, and is the "correct" definition for what is classically called the number of arbitrary functions of $m$ variables in the solution of the system $P=0(P \in \mathfrak{p})$. Moreover, if $\mathfrak{p}^{\prime}$ is another prime differential ideal of $a$ subject to the inclusion $\mathfrak{p} \subset \mathfrak{p}^{\prime}$ (or, equivalently, to the inclusion $\left.\mathbb{Z}(\mathfrak{p}) \supset \mathfrak{Z}\left(\mathfrak{p}^{\prime}\right)\right)$ then $d(\mathfrak{p}) \geqq d\left(\mathfrak{p}^{\prime}\right)$; however, when the inclusions are strict the inequality need not be so. This shows that $d(\mathfrak{p})$ is not a sufficiently fine measure of the size of $Z(p)$.

In what follows we present another measure, which is sufficiently fine, and describe its relation to $d(p)$ and some of its other properties; it is vaguely reminiscent of Hilbert's "characteristic function" for

\footnotetext{
1 This research was supported by the National Science Foundation.
} 
homogeneous polynomial ideals. ${ }^{2}$ For the sake of simplicity we continue to suppose that the differential field $\mathcal{F}$ is of characteristic 0 ; we denote the derivation operators of $\mathscr{F}$ by $\delta_{1}, \cdots, \delta_{m}$. We omit the proofs. All details, as well as generalization to nonzero characteristic, will appear in a book now in preparation.

We recall that a polynomial $f \in R[X]$ in one indeterminate is said to be numerical if $f(s) \in \boldsymbol{Z}$ for all sufficiently big $s \in N$. Any $f$ can be written in the form

$$
f=\sum_{k} a_{k}\left(\begin{array}{c}
X+k \\
k
\end{array}\right)
$$

where $a_{k} \in R$ and

$$
\left(\begin{array}{c}
X+k \\
k
\end{array}\right)=(X+1)(X+2) \cdots(X+k) / k !
$$

$f$ is numerical if and only if $a_{k} \in \boldsymbol{Z}$ for every $k .^{3}$ We define $f \leqq g$ to mean that $f(s) \leqq g(s)$ for all sufficiently big $s \in N$; this totally orders $R[X]$, and well orders the set of all numerical polynomials which are $\geqq 0$.

For any $s \in N$ there are

$$
n\left(\begin{array}{c}
s+m \\
m
\end{array}\right)
$$

derivatives $\delta_{1}^{i_{1}} \cdots \delta_{m}^{i_{m}} y_{j}$ with $i_{1}+\cdots+i_{m} \leqq s$ and $1 \leqq j \leqq n$; they may be regarded as indeterminates over the field $F$, and therefore the ring

$$
\mathfrak{Q}_{s}=\mathfrak{F}\left[\left(\delta_{1}^{i_{1}} \cdots \delta_{m}^{i_{m}} y_{j}\right)_{i_{1}+\cdots+i_{m} \leqq s, 1 \leqq j}\right]_{n}
$$

is a polynomial algebra over $\mathcal{F}$ in the usual sense. For any prime differential ideal $\mathfrak{p}$ of $\mathfrak{Q}, \mathfrak{p} \cap \mathfrak{Q}_{s}$ is a prime ideal of $\mathfrak{Q}_{s}$ and hence has a dimension.

Theorem 1. Let $\mathfrak{p}$ be a prime differential ideal of $a$. There exists a unique numerical polynomial $\omega_{\mathfrak{p}}$ such that $\operatorname{dim}\left(\mathfrak{p} \cap \mathfrak{Q}_{s}\right)=\omega_{\mathfrak{p}}(s)$ for all sufficiently big $s \in N$.

${ }^{2}$ For a general discussion of the characteristic function see e.g., [2, pp. 230-237]. By using a filtration instead of a grading one can equally well introduce the characteristic function in the nonhomogeneous theory.

${ }^{3}$ See e.g., $[2$, p. 233]. 
We call $\omega_{\mathfrak{p}}$ the differential dimension polynomial of $\mathfrak{p}$. Some of its properties are given by the following result.

Theorem 2. Let $\mathfrak{p}$ be a prime differential ideal of $a$.

$$
0 \leqq \omega_{\mathfrak{p}} \leqq n\left(\begin{array}{c}
X+m \\
m
\end{array}\right)
$$

so that $\operatorname{deg} \omega_{\mathfrak{p}} \leqq m$.

(b) $\omega_{\mathfrak{p}}=0$ if and only if $Z(\mathfrak{p})$ is a finite set.

(c) $\omega_{p}=n\left(\begin{array}{c}X+m \\ m\end{array}\right)$ if and only if $\mathfrak{p}=(0)$.

(d) If we write $\omega_{\mathfrak{p}}=\sum_{0 \leqq k \leqq n} a_{k}(\mathfrak{p})\left(\begin{array}{c}X+m \\ m\end{array}\right)$ then $a_{m}(\mathfrak{p})=d(\mathfrak{p})$.

Thus, $\omega_{p}$ contains at least as much information about $p$ as $d(p)$ does. The following observation shows that $\omega_{\mathfrak{p}}$ gives an adequate measure of the size of $Z(p)$.

TheOREm 3. Let $\mathfrak{p}, \mathfrak{p}^{\prime}$ be prime differential ideals of $\mathfrak{a}$ with $\mathfrak{p} \subset \mathfrak{p}^{\prime}$, $p \neq p^{\prime}$. Then $\omega_{p}>\omega_{p^{\prime}}$.

We recall (see [1, pp. 30-31 and 166-167]) that for an irreducible differential polynomial $P \in Q$ Ritt defined the notions of general component and singular component of $P$; precisely one of the components of $P$ is general, the rest all are singular.

TheOREM 4. Let $p$ be a prime differential ideal of a. A necessary and sufficient condition that $\mathfrak{p}$ be the general component of an irreducible differential polynomial in a of order $e$ is that

$$
\omega_{\mathfrak{p}}=n\left(\begin{array}{c}
X+m \\
m
\end{array}\right)-\left(\begin{array}{c}
X+m-e \\
m
\end{array}\right) .
$$

It follows that when this is the case then

$$
\omega_{p}=(n-1)\left(\begin{array}{c}
X+m \\
m
\end{array}\right)+e\left(\begin{array}{c}
X+m-1 \\
m-1
\end{array}\right)+\cdots,
$$

that is, $a_{m}(\mathfrak{p})=n-1$ and $a_{m-1}(\mathfrak{p})=e$.

We remark that $\omega_{p}$ is a birational invariant but not a differential birational invariant. By this we mean that if $\mathfrak{p}, \mathfrak{q}$ are prime differential ideals of $Q$ with respective generic zeros $\eta, \zeta$ then the condition 
$\mathscr{F}(\eta)=\mathscr{F}(\zeta)$ implies that $\omega_{p}=\omega_{q}$ but the weaker condition $\mathscr{F}\langle\eta\rangle=\mathscr{F}\langle\zeta\rangle$ does not. Nevertheless, $\omega_{p}$ carries with it certain differential birational invariants. One example is $a_{m}(\mathfrak{p})$. The following result provides others.

Theorem 5. For each prime differential ideal $\mathfrak{p}$ of $a$ with $\omega_{p} \neq 0$ set $m^{\prime}(\mathfrak{p})=\operatorname{deg} \omega_{\mathfrak{p}}$ and $d^{\prime}(\mathfrak{p})=a_{m^{\prime}(\mathfrak{p})}(\mathfrak{p})$. Then $m^{\prime}(\mathfrak{p})$ and $d^{\prime}(\mathfrak{p})$ are differential birational invariants.

We call $m^{\prime}(\mathfrak{p})$ the differential type of $\mathfrak{p}$ and call $d^{\prime}(\mathfrak{p})$ the typical differential dimension of $\mathfrak{p}$. The following result interprets these invariants and justifies the terminology somewhat.

Theorem 6. Let $\mathfrak{p}$ be a prime differential ideal of a having differential type $m^{\prime}$ and typical differential dimension $d^{\prime}$; let $\eta$ be a generic zero of $p$ and set $\mathcal{G}=\mathscr{F}\langle\eta\rangle$. Then there exists an $m^{\prime} \times m$ matrix $\left(c_{i^{\prime} i}\right)$ over the field of constants $\mathcal{C}$ of $\mathcal{F}$ of rank $m^{\prime}$ such that if we set $\delta_{i^{\prime}}^{\prime}=\sum_{1 \leqq i \leqq m} c_{i^{\prime} i} \delta_{i}$ $\left(1 \leqq i^{\prime} \leqq m^{\prime}\right)$ and regard $\mathcal{F}$ and $g$ as differential fields with the $m^{\prime}$ derivation operators $\delta_{1}^{\prime}, \cdots, \delta_{m^{\prime}}^{\prime}$ then $\mathrm{g}$ is a finitely generated differential field extension of $\mathcal{F}$ of differential transcendence degree $d^{\prime}$. The matrices $\left(c_{i^{\prime} i}\right)$ having this property form an open subset of $\mathfrak{e}^{m^{\prime} m}$ in the Zariski $\mathrm{C}$ topology.

In the classical terminology we could say that the solution of the system $P=0(P \in p)$ depends on $d^{\prime}$ arbitrary functions of $m^{\prime}$ variables (but not on any arbitrary functions of more than $m^{\prime}$ variables).

It would be interesting to find other differential birational invariants.

\section{BIBLIOGRAPHY}

1. J. F. Ritt, Differential algebra, Amer. Math. Soc. Colloq. Publ. Vol. 33, Amer. Math. Soc., Providence, R. I., 1950.

2. Oscar Zariski and Pierre Samuel, Commutative algebra, Vol. II, Van Nostrand, Princeton, N. J., 1960.

Columbia University 